\title{
Evaluation of an Unmanned Aerial System for Antenna Diagnostics and Characterization
}

\author{
Maria Garcia-Fernandez, Yuri Alvarez-Lopez, Fernando Las Heras \\ Area of Signal Theory and Communications, University of Oviedo, Gijón, Spain, mariagarcia@ @sc.uniovi.es
}

\begin{abstract}
The main goal of this contribution is to analyze the performance of an Unmanned Aerial System for Antenna Measurement (UASAM). UASAM can be used for performing antenna diagnostics and characterization at the antenna operational location. The proposed system measures the power transmitted by the Antenna Under Test (AUT) in the near field region. These measurements are post-processed to perform antenna diagnostics and to retrieve the far field pattern. Due to the lack of phase data, a phase retrieval technique called phaseless Sources Reconstruction Method (pSRM) is used to obtain the aperture fields. In this contribution, the effect of acquisition grid truncation in the aperture field reconstruction quality and in the far field patterns is studied. Three different grids have been considered: cylindrical and arc cylindrical surfaces, and planar surfaces parallel to the AUT aperture. It has been found that using planar acquisition grids the quality of the antenna diagnostics slightly improves and the flight times are shortened (compared to cylindrical acquisitions).
\end{abstract}

Index Terms-antenna measurement, Unmanned Aerial Vehicles (UAVs), phaseless Sources Reconstruction Method (SRM), Near-Field to Far-Field transformation (NF-FF).

\section{INTRODUCTION}

In the last years, several systems based on the use of Unmanned Aerial Vehicles (UAVs) have been proposed for antenna and electromagnetic emissions measurement. Despite being less accurate than anechoic chambers or outdoor ranges measurements, they allow the measurement of the radiation pattern in operational conditions. This implies that they take into account how the environment affects the radiation pattern (for instance, reflections at the ground surface). They are also less expensive than other systems for the similar purposes such as those based on manned aircrafts.

Most UAV-based antenna measurement systems make use of a power detector [1], a spectrum analyzer [2] or a radiofrequency transmitter [3] onboard. In the latter, the Antenna Under Test (AUT) works as a receiver. These systems operate at the Far-Field (FF) region of the AUTs, measuring the radiation pattern directly. It must be noticed that the FF region can be hundreds of meters away from the AUT for low frequency and/or electrically large antennas. As a result, one advantage of these systems is that they are not so sensitive to the UAV positioning errors. However, there could be practical limitations on the usage of these systems mainly due to the long flight time needed for the whole radiation pattern acquisition (which might require several flights due to the small flight autonomy of most UAVs) and the flight regulations that may restrict the measurement region.
In order to deal with some of these limitations, an Unmanned Aerial System for Antenna Measurement (UASAM) operating in the Near-Field (NF) region up to $\mathrm{C}$ band has been developed [4][5]. From the NF measurements, a Near-Field to Near-Field (NF-NF) transformation is performed to recover the fields at the AUT aperture, which enables antenna diagnostics. Then, once an equivalent current distribution is reconstructed, a Near-Field to Far-Field (NF-FF) transformation is applied to retrieve the radiation pattern.

Furthermore, aiming to achieve a low cost and low complexity system, a power detector is used instead of a coherent receiver. Thus, since only amplitude measurements are available, a phase retrieval technique must be used to perform the NF-NF and NF-FF transformations. In particular, an iterative phase retrieval technique called phaseless Sources Reconstruction Method (pSRM) [6] is used instead of more complex techniques such as indirect holographic ones [7]. This technique requires the measurement of the NF on at least two acquisition surfaces since it takes advantage of the variation of the field distribution with the distance to estimate the phase.

In the validation of the initial prototype several antenna arrays were measured at two concentric cylindrical surfaces, requiring in some cases more than one flight to acquire all the data. Therefore, in this contribution, the truncation of the acquisition domain (from two concentric cylindrical surfaces to two planar surfaces or two cylindrical arc surfaces) is analyzed by measuring a 2-element horn antenna array at 4.65 GHz. The main goal is to achieve a good balance between measurement accuracy and acquisition domain size (which is directly related to flight time).

\section{DESCRIPTION OF THE PROPOSED SYSTEM}

The first prototype of the proposed system is composed by the following subsystems:

- Flight control subsystem, which contains the flight controller, the communication devices and the usual positioning sensors. These sensors are: Inertial Measurement Units (IMUs), barometer and Global Navigation Satellite Systems (GNSSs).

- Accurate positioning subsystem, which makes use of a laser rangefinder and a Real Time Kinematic (RTK) system. The RTK system consists of two RTK beacons: one onboard the UAV and the other at a fixed position (working as base station and sending GNSS corrections to the rover beacon). 


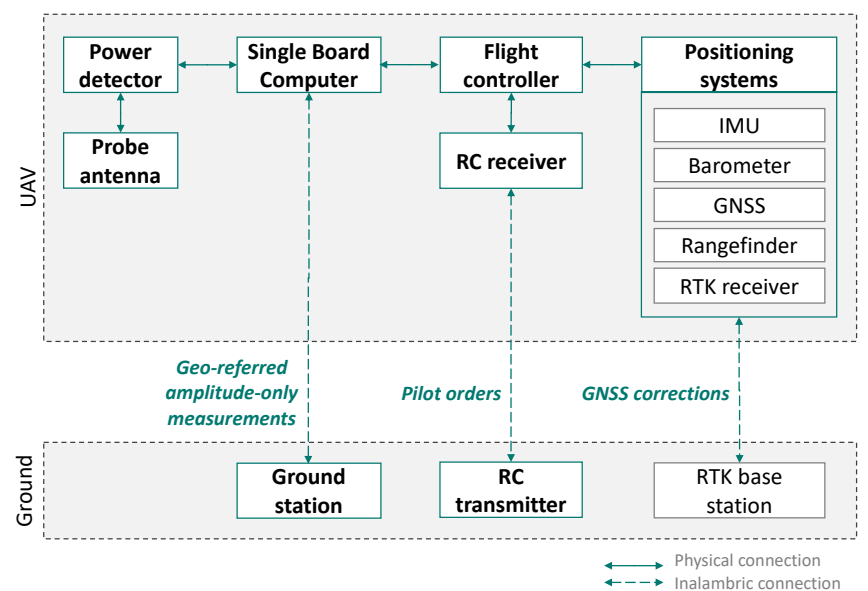

Fig. 1. Scheme of the UASAM prototype: main components and connections.

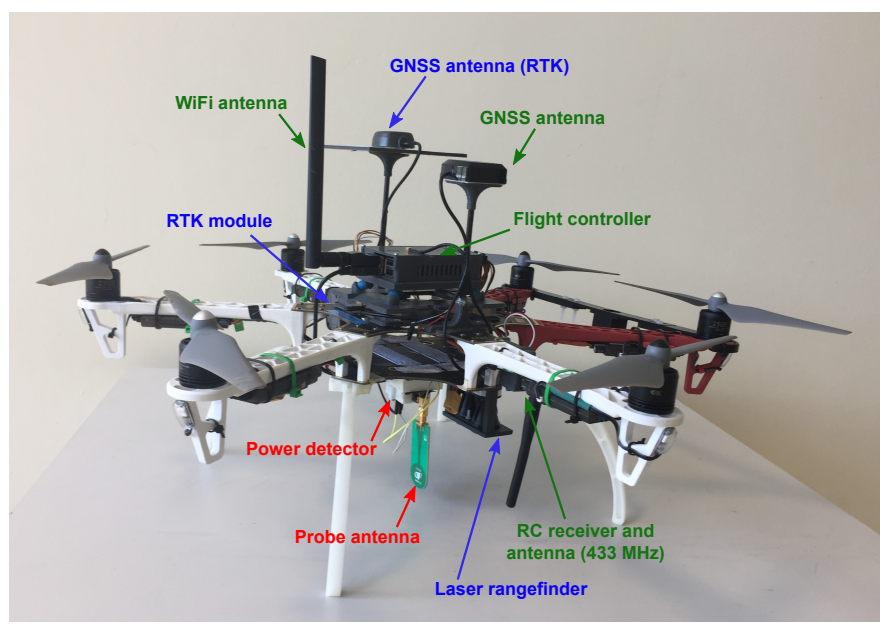

Fig. 2. UASAM prototype: flight control subsystem in green, accurate positioning subsystem in blue and antenna measurement subsystem in red.

- Antenna measurement subsystem, which includes a power detector and a probe antenna.

- Ground control station where the geo-referred amplitudeonly measurements are post-processed.

An scheme of the main prototype components together with the connections between them is depicted in Fig. 1. The prototype used in this contribution is shown in Fig. 2.

Prior to the flight, a flight plan is set using waypoints. This flight plan describes the measurement grid which, in this case, is composed by two concentric cylinders, two concentric cylindrical arc surfaces or two parallel planes. It must be noticed that in the first two cases the UAV heading points to the AUT (as in cylindrical measurement ranges), whereas in the later the heading is perpendicular to the measurement planes. The UAV coordinates and the measurements of the power detector are sent to the ground station, where they are post-processed to perform antenna diagnostics and to obtain the radiation pattern.

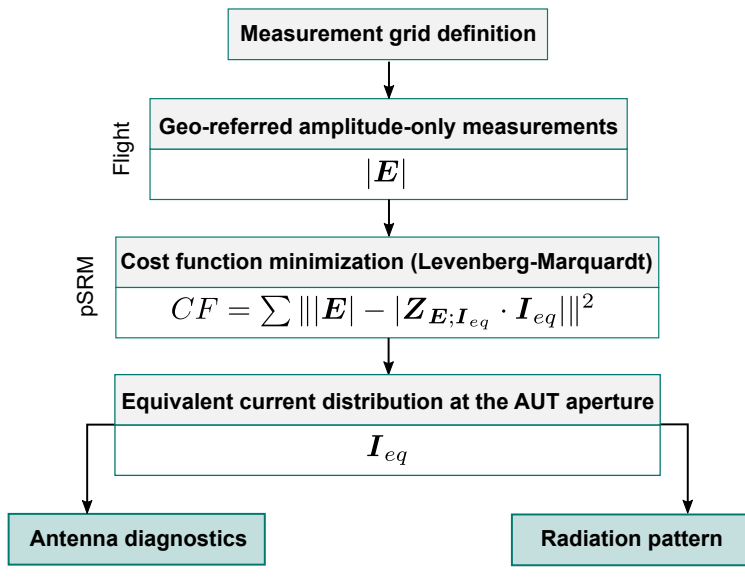

Fig. 3. Scheme of the measurements acquisition and post-processing steps.

\section{MEthodology}

As aforementioned, since the prototype makes use of a power detector, phase cannot be measured and a phase retrieval method must be applied. The phase retrieval method used in this contribution (pSRM) reconstructs an equivalent electric and magnetic currents distribution on a surface enclosing the AUT (in practice, at the AUT aperture). With this distribution we can perform antenna diagnostics as well as computing the radiation pattern with a NF-FF transformation.

Fig. 3 shows the workflow of the proposed antenna measurement method. As shown there, the pSRM tries to minimize a non-linear cost function that takes into account the difference between the measured amplitude $(|\mathbf{E}|)$ and the amplitude of the field radiated by an equivalent currents distribution $\left(\mathbf{I}_{e q}\right)$. Due to the lack of phase data, it requires to acquire the measurements at two different acquisition surfaces. The main advantage of this method is that it can handle arbitrary measurement grids as long as the sampling rate is smaller than $\lambda / 4$ (where $\lambda$ is the wavelength).

\section{SETUP DESCRIPTION}

\section{A. AUT and antenna probe}

The AUT is a two horn antenna array working at $\mathrm{C}$ band (from 4 to $6 \mathrm{GHz}$ ). It is fed with a Voltage Controller Oscillator (VCO) and a RF amplifier, which provide a $+10 \mathrm{dBm}$ signal at $4.65 \mathrm{GHz}$. The AUT is placed in a mast at $3 \mathrm{~m}$ height and the distance between the horns is approximately $4 \lambda$. The measurement setup is shown in Fig. 4.

The antenna probe is a commercial printed monopole working from 4 to $7 \mathrm{GHz}$ with vertical polarization and an omnidirectional pattern in azimuth $( \pm 1.5 \mathrm{~dB}$ accuracy) [8].

\section{B. Measurement grids}

Measurement grids are defined in a flight plan (composed of waypoints) that the UAV autonomously tries to follow. The grids evaluated in this contribution are depicted in Figs. 57. In these grids, the dashed lines represent the pre-defined flight paths and the dots are the waypoints. It must be noticed 


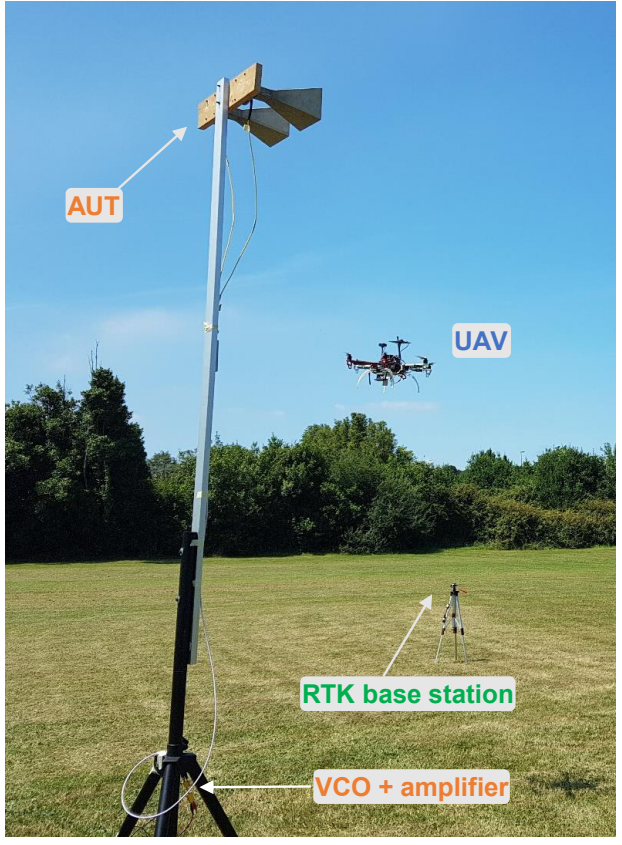

Fig. 4. UASAM measurement setup. The AUT is a 2-horn antenna array.

that the distance from the AUT has been increased in grids \#2 and \#3 for safety reasons. As mentioned before, for the cylindrical grids the UAV heading points always towards the AUT, whereas in the planar grid the UAV heading is perpendicular to the AUT aperture.

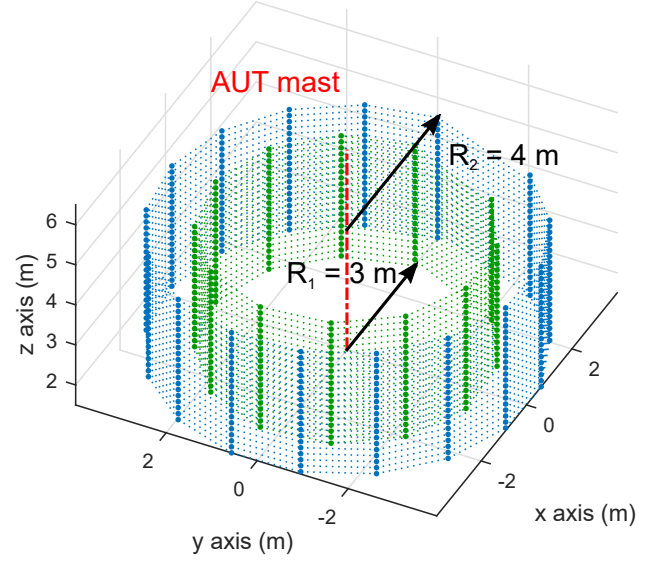

Fig. 5. Grid \#1: cylindrical grid of radius $R_{1}=3 \mathrm{~m}$ and $R_{2}=4 \mathrm{~m}$, with the height going from 1.5 to $4.5 \mathrm{~m}$ in $0.15 \mathrm{~m}$ steps.

\section{Comparison flowchart}

Antenna diagnostics and the far field radiation patterns obtained with UASAM have been compared with those obtained from measurements in anechoic chamber. Furthermore, the NF amplitude at the UAV positions has been also simulated in order to analyze the influence of positioning and measurement errors. A flowchart of these comparisons is shown in Fig. 8.

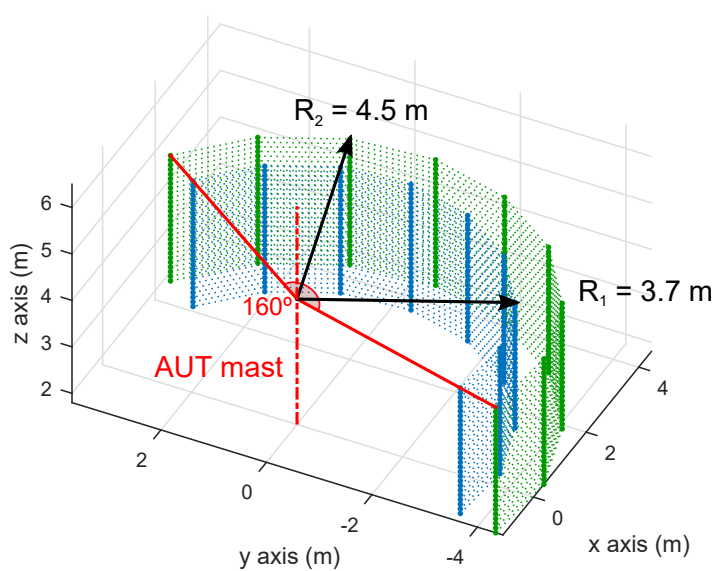

Fig. 6. Grid \#2: arc cylindrical surfaces of radius $R_{1}=3.7 \mathrm{~m}$ and $R_{2}=4.5$ $\mathrm{m}$, with the height going from 2 to $4.5 \mathrm{~m}$ in $0.1 \mathrm{~m}$ steps.

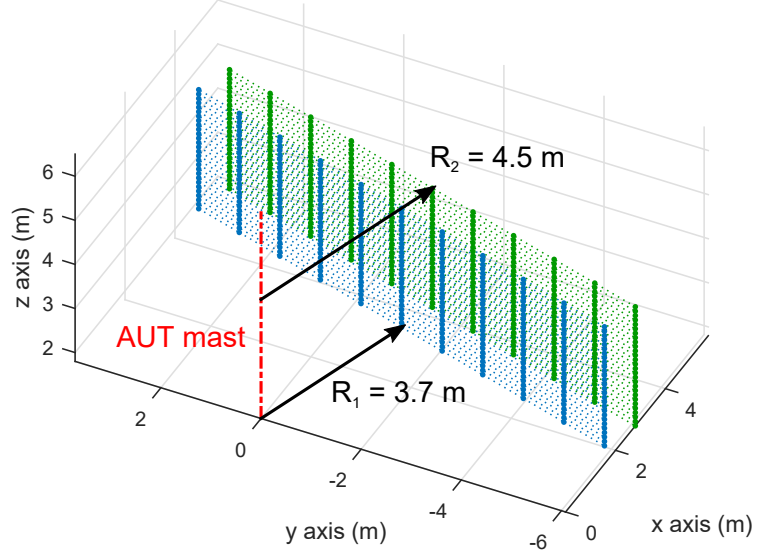

Fig. 7. Grid \#3: parallel planes of $10 \mathrm{~m}$ width at $R_{1}=3.7 \mathrm{~m}$ and $R_{2}=4.5$ $\mathrm{m}$, with the height going from 1.8 to $4.5 \mathrm{~m}$ in $0.1 \mathrm{~m}$ steps.

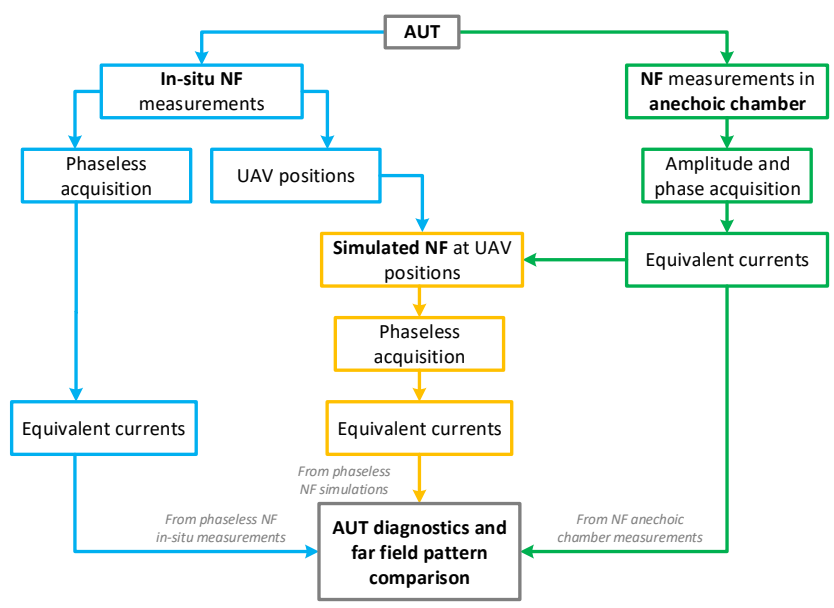

Fig. 8. Flowchart for comparing measurements accuracy. 


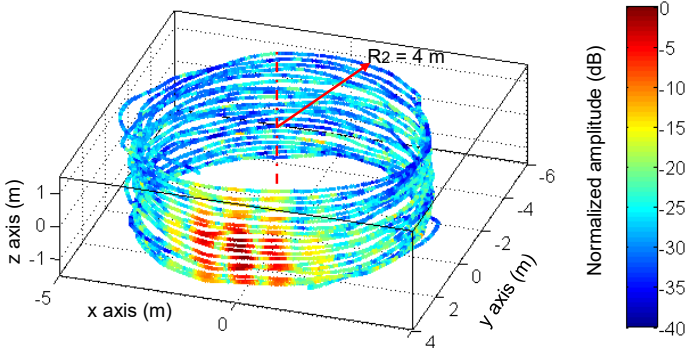

Fig. 9. Measured NF amplitude for grid $\# 1$ at $R_{2}=4 \mathrm{~m}$ radius.

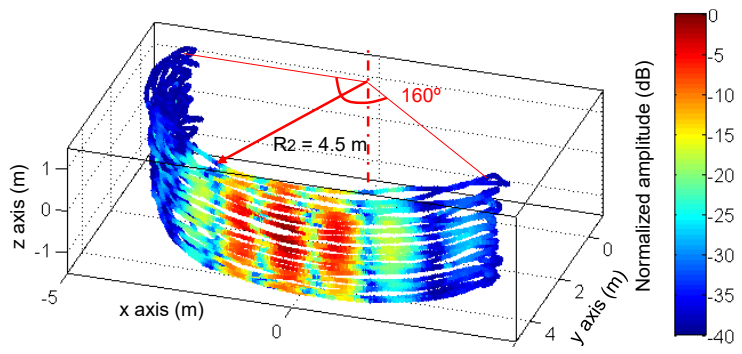

Fig. 10. Measured NF amplitude for grid \#2 at $R_{2}=4.5$ m radius.

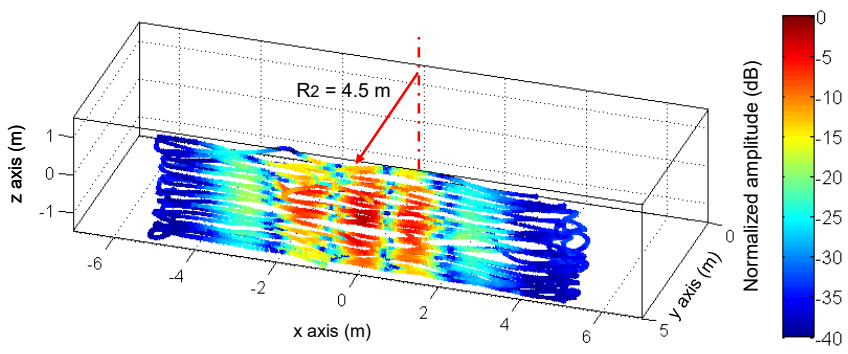

Fig. 11. Measured NF amplitude for grid \#3 at $R_{2}=4.5 \mathrm{~m}$ radius.

\section{EXPERIMENTAL VALIDATION}

\section{A. Near field measurements}

The measured NF amplitude of the AUT is shown for each grid in Figs. 9-11 (in particular, for the surface at distance or radius $R_{2}$ of each grid). In these figures the axes are centered at the AUT position. It has been observed that probe antenna misalignments are more noticeable in planar measurement grids (i.e. in grid \#3). In addition, these misalignments increase as the acquisition plane gets closer to the AUT.

The flight times for each grid are approximately: 25 minutes for grid \#1, 20 minutes for grid \#2, and 17 minutes for grid $\# 3$. Notice that the flight time of grid \#1 would have been even longer if the measurements had been taken at a grid with the same radius as grid $\# 2$.

\section{B. Antenna diagnostics}

The geo-referred amplitude-only NF measurements are post-processed using pSRM. The reconstruction domain on the AUT aperture plane, where the currents distribution is recovered, is a rectangular domain of $60 \times 60 \mathrm{~cm}$. This domain must be large enough to contain the projected physical AUT aperture, but not too large in order to keep the number of equations and the problem conditioning as low as possible.

In Fig. 12 the recovered equivalent currents distribution is shown for the following cases: NF amplitude and phase measurements at anechoic chamber (a), simulated NF amplitude at UAV positions for grid \#1 (b), and NF amplitude measurements with UASAM for grids \#1 (c), \#2 (d) and \#3 (e). In all cases the location of each antenna as well as its relative emitted power can be estimated.

Comparing cases (a) and (b), it can be concluded that, as expected, the use of amplitude-only measurements and the deviations of the UAV from the ideal flight path worsen the quality of the reconstructed currents.

Regarding cases (b) and (c), the effects of geo-referring errors and probe antenna misalignments in the reconstruction quality can be observed.

When the grid is composed of arc cylindrical surfaces (d), the reconstruction is even slightly worse due to the truncation of the measurement grid (even though the AUT is directive) and due to the fact that the UAV stops at each arc end point to change direction. The latter implies that the UAV deviations from the ideal path are slightly increased.

Finally, when a planar grid is considered we obtain the best reconstruction quality (e). This might be due to the fact that the UAV heading does not change (it is always perpendicular to the AUT), whereas in cylindrical grids the UAV heading is continuously changing so as to point to the AUT position. However, it must be noticed that it requires to accurately determine the direction perpendicular to the AUT and it can only be used when measuring directive antennas.

\section{Far field pattern}

The recovered equivalent currents distributions at the AUT aperture are further post-processed to obtain the radiation pattern. As shown in Fig. 13 there is a good agreement between the reference FF pattern and those measured with UASAM, especially for the main lobes $\left(\theta \in\left[-20^{\circ}, 20^{\circ}\right]\right)$. It must be pointed out that the discrepancies are mainly due to the geo-referring errors (which are around $2 \mathrm{~cm}$ in the horizontal plane and $1 \mathrm{~cm}$ in the vertical direction).

\section{CONCLUSION}

In this contribution, the use of UASAM for measuring antennas in their operational location is analyzed. In particular, UASAM is tested for measuring a two-element horn antenna array working at $\mathrm{C}$ band using three different acquisition grids. It has been shown that measuring at two complete cylindrical surfaces requires the longest flight time. Truncating it to arc cylindrical surfaces $\left(160^{\circ}\right)$ degrades the quality of the reconstructed equivalent currents distribution but the flight time is not greatly reduced. However, using planar measurement surfaces does not degrade the quality (as compared to cylindrical surfaces) and it takes around $35 \%$ less time. In this case, the main challenges are due to the increase of probe antenna misalignments and the need of accurately knowing the direction perpendicular to the AUT surface. 

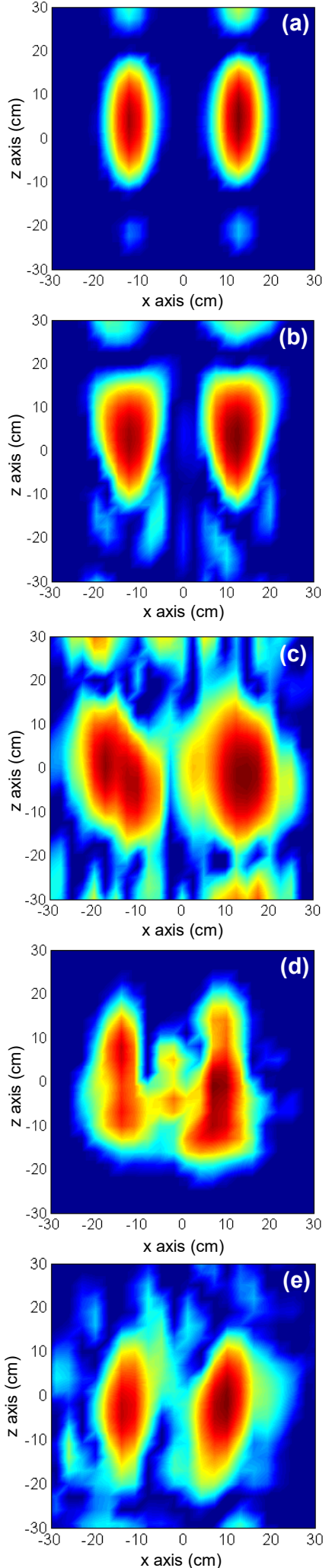

Fig. 12. Recovered aperture fields (equivalent currents distribution) at the AUT aperture: NF measurements at anechoic chamber (a), simulated NF amplitude at UAV positions for grid \#1 (b) and NF amplitude measurements with UASAM for grids \#1 (c), \#2 (d) and \#3 (e)

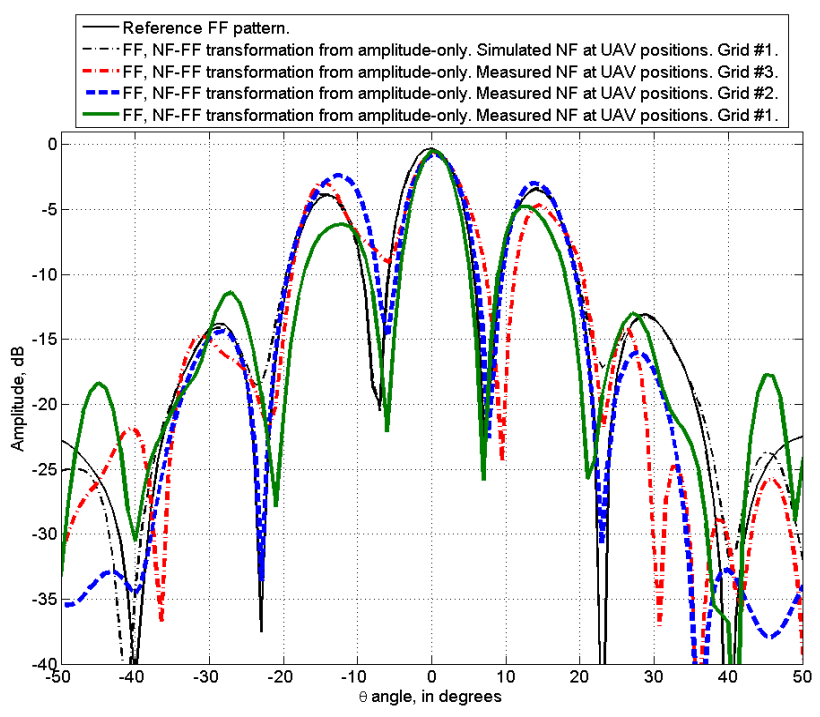

Fig. 13. FF patterns obtained from the retrieved equivalent currents $(\mathrm{H}$-plane $\phi=0^{\circ}$ ). Amplitude is normalized to the maximum of each 3D FF pattern.

\section{ACKNOWLEDGMENT}

This work has been partially supported by the "Ministerio de Economía y Competitividad" of Spain/FEDER under projects TEC2014-55290-JIN (PORTEMVISION) and TEC2014-54005-P (MIRIIEM); by the "Ministerio of Educación y Cultura" of Spain under FPU grant FPU15/06341; and by the Government of Asturias under project GRUPIN 14-114.

\section{REFERENCES}

[1] M. S. Sharawi, "Unmanned aerial vehicle for antenna radiation characterization," US Patent Appl. US 20160088498 A1, published March 2016.

[2] J. Schreiber, "Antenna pattern reconstitution using unmanned aerial vehicles (UAVs)," in IEEE Conference on Antenna Measurements and Applications (CAMA), Syracuse (USA), October 2016.

[3] G. Virone, A. M. Lingua, M. Piras, A. Cina, F. Perini, J. Monari, F. Paonessa, O. A. Peverini, G. Addamo, R. Tascone, "Antenna pattern verification system based on a micro unmanned aerial vehicle (UAV)," IEEE Antenn. Wireless Propag. Lett., vol. 13, pp. 169-172, 2014.

[4] M. Garcia-Fernandez, Y. Alvarez-Lopez, A. Arboleya, B. Gonzalez Valdes, Y. Rodriguez-Vaqueiro, M. E. de Cos, F. Las Heras, "Antenna Diagnostics and Characterization using Unmanned Aerial Vehicles," IEEE Access, accepted for publication, 2017.

[5] Y. Alvarez-Lopez, M. Garcia-Fernandez, F. Las Heras, A. ArboleyaArboleya, B. Gonzalez-Valdes, Y. Rodriguez-Vaqueiro, A. Garcia-Pino, "Sistema aerotransportado y método para la caracterización y medida de antenas o sistemas radiantes," Spanish Patent Appl. P201700209, 15-032017.

[6] Y. Alvarez, F. Las-Heras, M. R Pino, "The sources reconstruction method for amplitude-only field measurements," IEEE Trans. Antenn. Propag., vol. 58 , no. 8, pp. 2776-2781, 2010.

[7] J. Laviada, A. Arboleya, Y. Alvarez, C. Garcia, F. Las-Heras, "Phaseless antenna diagnostics based on off-axis holography with synthetic reference wave, IEEE Antenn. Wireless Propag. Lett., vol. 13, pp. 43-46, 2014.

[8] BroadSpec UWB Antenna Datasheet of Time Domain http://www.timedomain.com/datasheets/TD_Broadspec Antenna.pdf. Last access: 1/09/2017. 